\title{
On the generation mechanism of terminator times in subionospheric VLF/LF propagation and its possible application to seismogenic effects
}

\author{
M. Yoshida, T. Yamauchi, T. Horie, and M. Hayakawa \\ Department of Electronic Engineering and Research Station on Seismo Electromagnetics, University of \\ Electro-Communications, 1-5-1 Chofugaoka, Chofu Tokyo 182-8585, Japan
}

Received: 29 November 2007 - Revised: 22 January 2008 - Accepted: 22 January 2008 - Published: 25 February 2008

\begin{abstract}
The signals from VLF/LF transmitters are known to propagate in the Earth-ionosphere waveguide, so that the subionospheric propagation characteristics are very sensitive to the condition of the lower ionosphere. We know that there appear the terminator times just around the sunrise and sunset in the diurnal variation of subionospheric VLF/LF signal (amplitude and phase). These terminator times are found to shift significantly just around an earthquake, which enables us to infer the change in the ionosphere during the earthquake. In this paper we try to understand the physical mechanism on the generation of those terminator times for relatively short propagation path (less than $2000 \mathrm{~km}$ ) by means of wave-hop method. It is found that the lowering of the ionosphere boundary during an earthquake decreases the path length of the sky wave and this alters the interference condition of this wave with the ground wave, which lead to an appearance of terminator times as the destructive interference between the ground and sky waves. Finally, we suggest a possible use of terminator time shifts to investigate the lower ionospheric plasma changes during the earthquakes.
\end{abstract}

\section{Introduction}

Extensive studies have been recently performed on the possible use of electromagnetic effects in the earthquake prediction (Hayakawa and Fujinawa, 1994; Hayakawa, 1999; Hayakawa and Molchanov, 2002). Among different electromagnetic effects, the detection of seismo-ionospheric VLF/LF propagation anomaly, has attracted a lot of attention. Since VLF/LF signals propagate in the Earth-ionosphere waveguide, the amplitude (and/or phase) of the signal re-

Correspondence to: M. Hayakawa

(hayakawa@whistler.ee.uec.ac.jp) ceived at a station is highly influenced by plasma condition of the lower ionosphere. There have been proposed two methods to analyze such VLF/LF diurnal variations for the study of seismo-ionospheric perturbations; (1) terminator time method (Hayakawa et al., 1996; Molchanov and Hayakawa, 1990; Molchanov et al., 1998) and (2) nighttime fluctuation method (Shvets et al., 2004; Rozhnoi et al., 2004; Maekawa et al., 2006). In the former method, we pay attention to the terminator times in subionospheric VLF/LF diurnal variation, which are defined as the times of minimum in amplitude (or phase) around sunrise and sunset. These terminator times are found to shift significantly just around the earthquake. In the case of Kobe earthquake, Hayakawa et al. (1996) found significant shifts in both morning and evening terminator times and these authors interpreted the shift in terminator time in terms of the lowering of lower ionosphere by using the full-wave mode theory. In the latter method of nighttime fluctuation, we estimate the difference between the nighttime variation in amplitude (or phase) and the corresponding average (e.g. monthly average). By using this nighttime fluctuation method, Maekawa et al. (2006) have found that there are two significant effects in the nighttime ionosphere associated with earthquakes; (1) decrease in the average amplitude and (2) enhancement in the fluctuation.

In this paper, we pay attention to the former effect on the terminator time associated with earthquakes. By using the wave-hop method (unlike the previous method of mode theory in Hayakawa et al., 1996 and Molchanov et al., 1998), we try to interpret the generation mechanism of terminator times in subionospheric VLF/LF diurnal pattern in a physical way. Then, the possible use of the shift in terminator times is suggested to indicate the seismogenic effect in the lower ionosphere by using the relationship of possible terminator time shift to the change in the lower ionosphere.

Published by Copernicus Publications on behalf of the European Geosciences Union. 


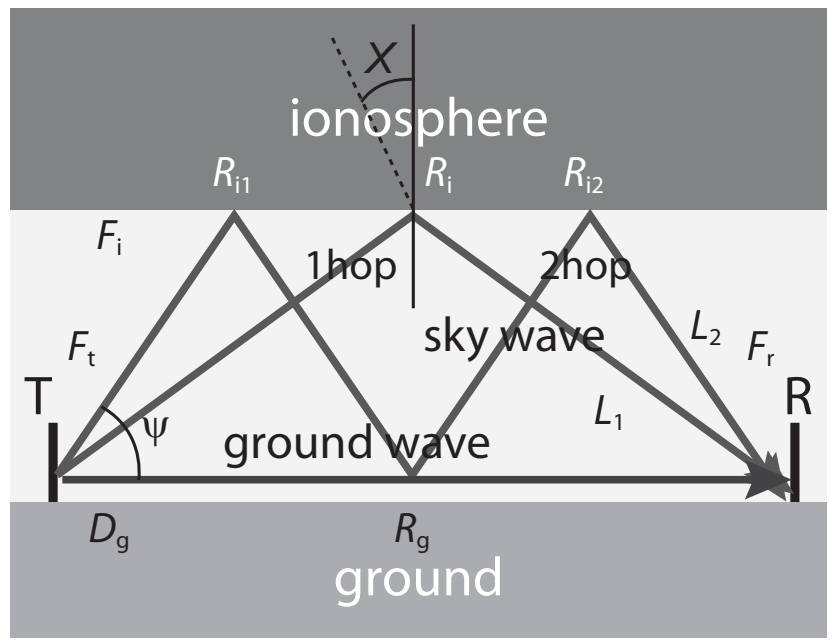

Fig. 1. VLF/LF propagation modeling (ground wave and sky waves).

\section{Subionospheric VLF/LF propagation in terms of wave-hop method}

The wave-hop method used in this paper is principally based on ray theory, but it takes into account the wave intensity as well as the wave ray-path computation. The wave-hop method is described briefly here. The wave received at a receiver is composed of ground wave and sky waves propagated in the Earth-ionosphere waveguide. The computation of sky waves is principally based on the ray theory, and the ground wave is calculated by the full-wave Sommerfeld integrations. More details are given in ITU $(1992,2002)$, Wakai et al. (2004) and its successful use has been given in Biagi et al. (2006). The configuration of our problem is given in Fig. 1, in which you can notice a 1-hop sky wave and a 2hop sky wave. Generally, we can include many higher-hop waves, such as n-hop wave (n: the number of reflection from the ionosphere). In the case of 1-hop wave, the reflection point of the ionosphere is located just in the middle between the transmitter and receiver. The reflection coefficient at the ionosphere is defined by $\mathrm{R}_{i}$. The ionospheric height is a function of the solar zenith angle $(\chi)$, so that the launching elevation angle $(\psi)$ and path length $(\mathrm{L})$ are determined by the distance between the transmitter and receiver (d) and ionospheric height (h). Though Fig. 1 is illustrated for a flat configuration, the real situation is for curved Earth and ionosphere, and this results in an additional effect of focussing expressed by $\mathrm{F}_{i}$ (focussing at the ionosphere). With taking all effects into account, the electric field at the receiving point is given by,

$\mathrm{E}_{1}=\frac{600 \sqrt{\mathrm{P}_{t}} \cos \psi \mathrm{R}_{i} \mathrm{~F}_{i} \mathrm{~F}_{t} \mathrm{~F}_{r}}{\mathrm{~L}_{1}}$

where $\mathrm{P}_{t}$ is the transmission power, $\mathrm{F}_{t}, \mathrm{~F}_{r}$ are transmitting and receiving antenna coefficients, respectively, which are

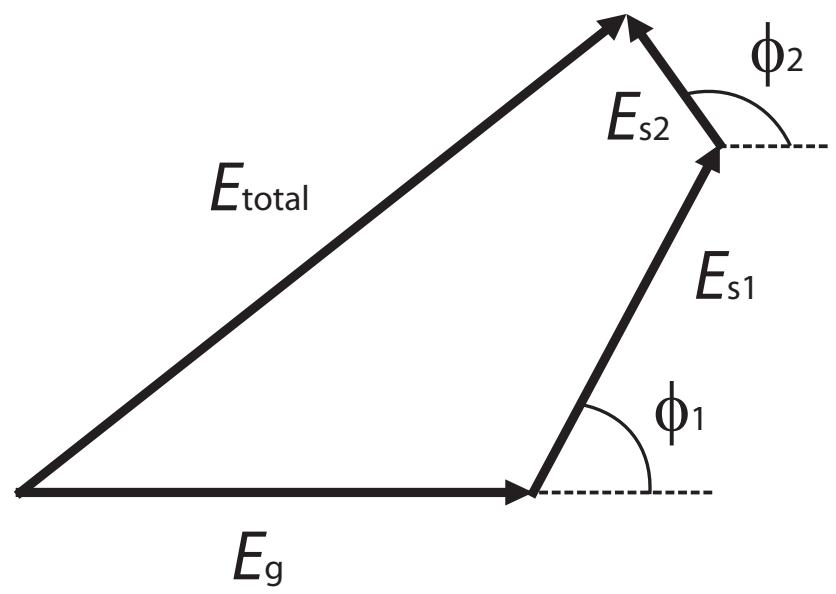

Fig. 2. Phasor diagram to have the resultant wave (as the superposition of ground wave and sky waves 1-hop and 2-hop waves). $\mathbf{E}_{\text {total }}$ is the total resultant wave, while $\mathbf{E}_{s 1}$ and $\mathbf{E}_{s 2}$ are the 1-hop and 2hop waves, with $\phi_{1}$ and $\phi_{2}$ being their phase delay with respect to the ground wave.

determined by the frequency and elevation angle $(\psi)$. These antenna coefficients are just those converting the electric field into the voltage. $L_{1}$ is the total path length.

When we consider a 2-hop wave, the wave suffers from the ionospheric reflections twice and one ground reflection. The reflection coefficients for this case are given by $\mathrm{R}_{i 1}, \mathrm{R}_{i 2}$, and $\mathrm{R}_{g}$. In contrast to the focussing at the ionosphere, we expect the divergence at the ground, which is expressed by another coefficient, $\mathrm{D}_{g} . \mathrm{L}_{2}$ is the total path length for this 2-hop wave. The electric field at the receiver is given by the following equation for the 2-hop wave.

$\mathrm{E}_{2}=\frac{600 \sqrt{\mathrm{P}_{t}} \cos \psi \mathrm{R}_{i 1} \mathrm{R}_{i 2} \mathrm{~F}_{i 1} \mathrm{~F}_{i 2} \mathrm{R}_{g} \mathrm{D}_{g} \mathrm{~F}_{t} \mathrm{~F}_{r}}{\mathrm{~L}_{2}}$

Generally speaking, the reflection and focussing coefficients of the ionosphere are not constant. On the assumption that the ionosphere is uniform over a relatively short distance, we assume that the ionosphere is uniform over a relatively short distance, and we assume that the ionospheric reflection coefficients are equal $\left(\mathrm{R}_{i 1}=\mathrm{R}_{i 2}\right)$ and constant and also the ionospheric height (h) is also constant. Further, we can assume the relationship of $\mathrm{F}_{i} \cdot \mathrm{D}_{g}=1$, and Eq. (2) is reduced to,

$\mathrm{E}_{2}=\frac{600 \sqrt{\mathrm{P}_{t}} \cos \psi \mathrm{R}_{i}^{2} \mathrm{~F}_{i} \mathrm{R}_{g} \mathrm{~F}_{t} \mathrm{~F}_{r}}{\mathrm{~L}_{2}}$

Generally the n-hop wave is also expressed by,

$\mathrm{E}_{n}=\frac{600 \sqrt{\mathrm{P}_{t}} \cos \psi \mathrm{R}_{i}^{n} \mathrm{~F}_{i} \mathrm{R}_{g}^{n-1} \mathrm{~F}_{t} \mathrm{~F}_{r}}{\mathrm{~L}_{n}}$

We have to sum up the 1 -hop, .... and n-hop waves to have a resultant field. In this paper we assume a relatively short 

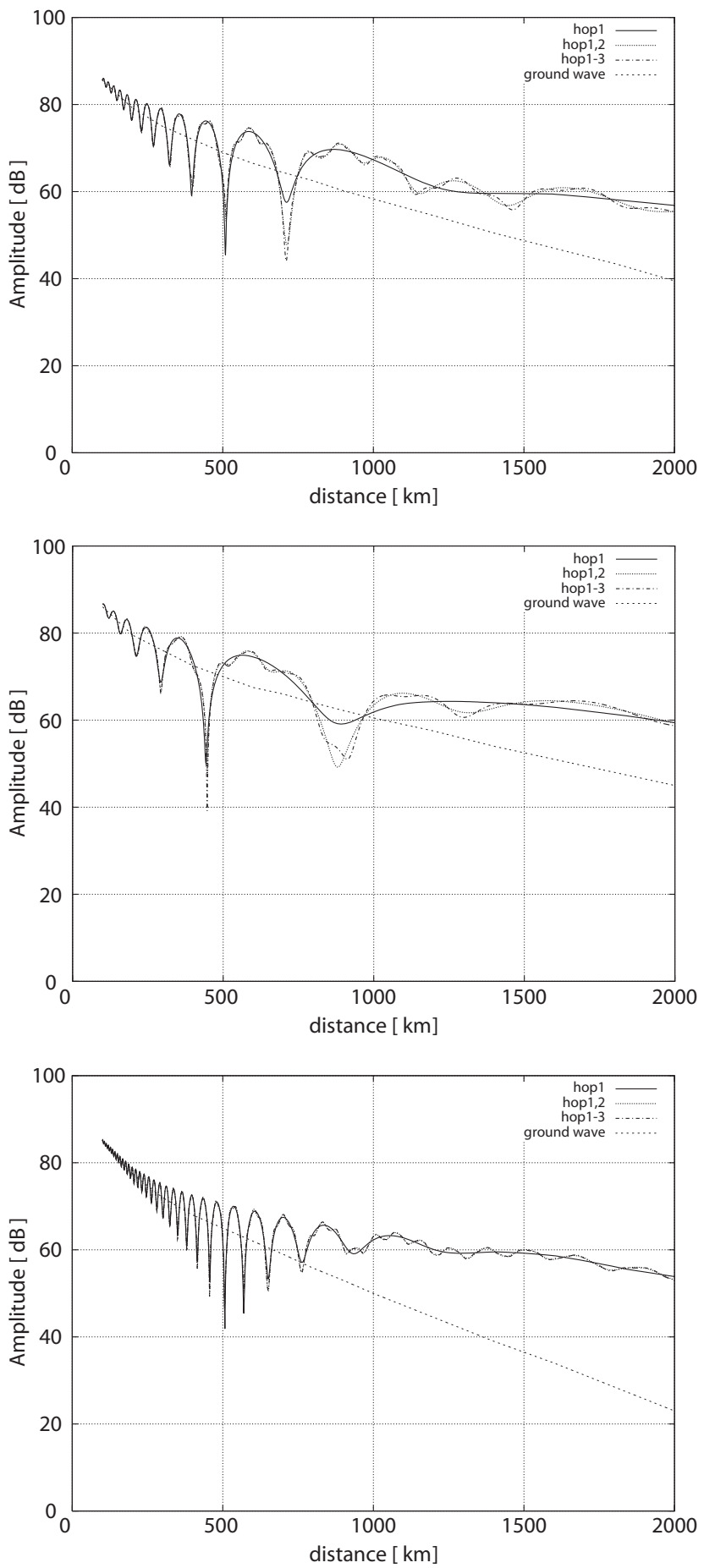

Fig. 3. Subionospheric electric field at the receiver as a function of propagation distance from the transmitter. (a) $40 \mathrm{kHz}$, (b) $20 \mathrm{kHz}$ and (c) $100 \mathrm{kHz}$.

propagation distance of $1000-2000 \mathrm{~km}$, so that it is found that we have to consider only up to a few-hop waves.

Figure 2 illustrates the phasor diagram, in which the total resultant wave ( $\left.\mathbf{E}_{\text {total }}\right)$ is a sum of the ground wave $\left(\mathbf{E}_{g}\right)$ and
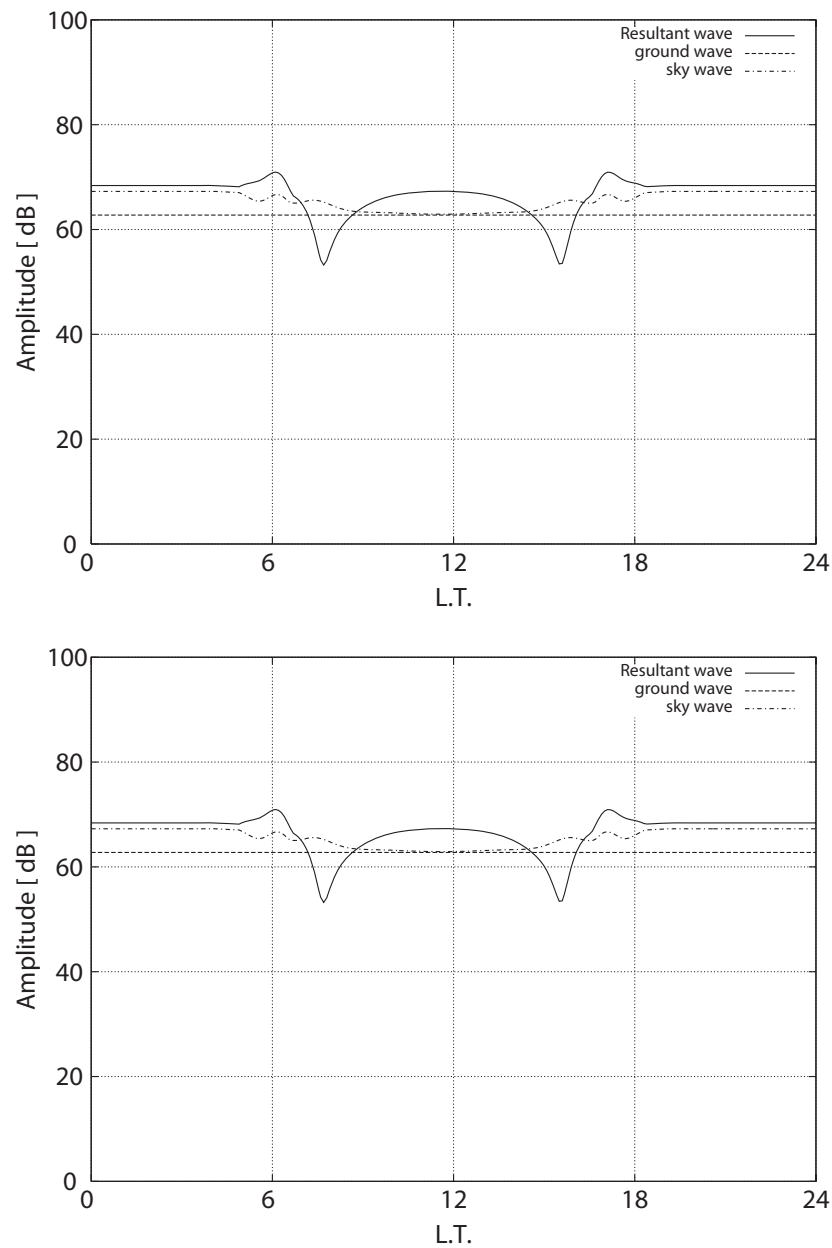

Fig. 4. (a) Diurnal variations of the electric field intensity for the ground wave (broken line), sky waves (dot-broken line) and resultant wave (full line) for the JJY-Kochi path. (b) Durnal variation of reflection height (full line) and phase delay of the 1-hop wave with respect to the ground wave $\left(\phi_{1}\right)$. The bottom is the resultant terminator time.

two sky waves (1-hop wave $\mathbf{E}_{s 1}$ and 2-hop wave $\mathbf{E}_{s 2}$ ). Here, $\phi_{1}$ and $\phi_{2}$ are their phase delays relative to the ground wave $\left(\mathbf{E}_{g}\right)$, which are resulted from the optical path difference and from the reflection at the iohosphere. However, when the propagation distance (d) is relatively small, the phase shift in the ionosphere is negligible, so that the phase delay of the sky wave with respect to the ground wave (L: total path length) is given by,

$\phi \cong \frac{2 \pi}{\lambda}(\mathrm{L}-\mathrm{d})$

\section{Computational results on terminator times}

Our Japanese VLF/LF network is consisted of seven receiving stations (Moshiri (Hokkaido), Chofu, Chiba (Tateyama), Shimizu, Kasugai, Maizuru, and Kochi). We observe, 


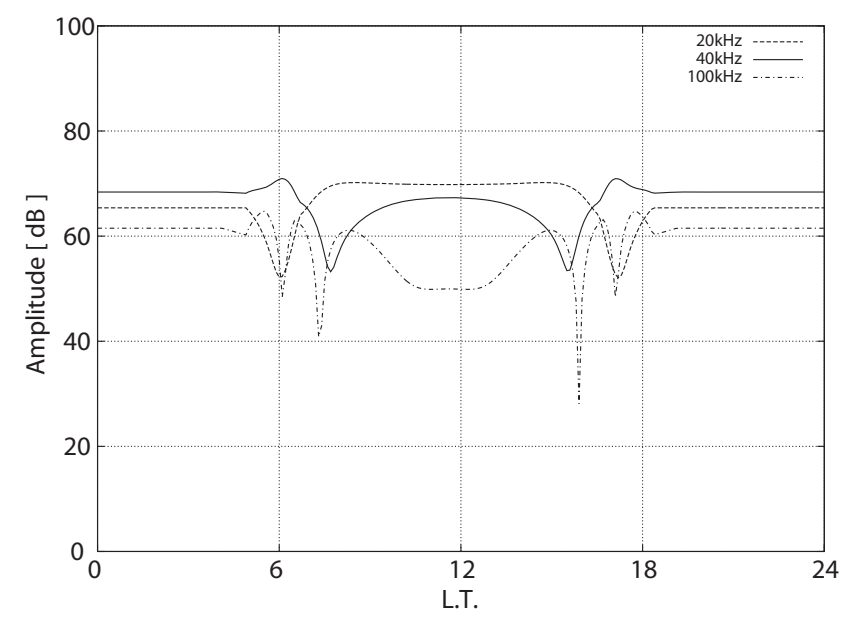

Fig. 5. Diurnal variation of the resultant electric field intensity for different frequencies $(20,40$ and $100 \mathrm{kHz})$ for a fixed distance of $780 \mathrm{~km}$.

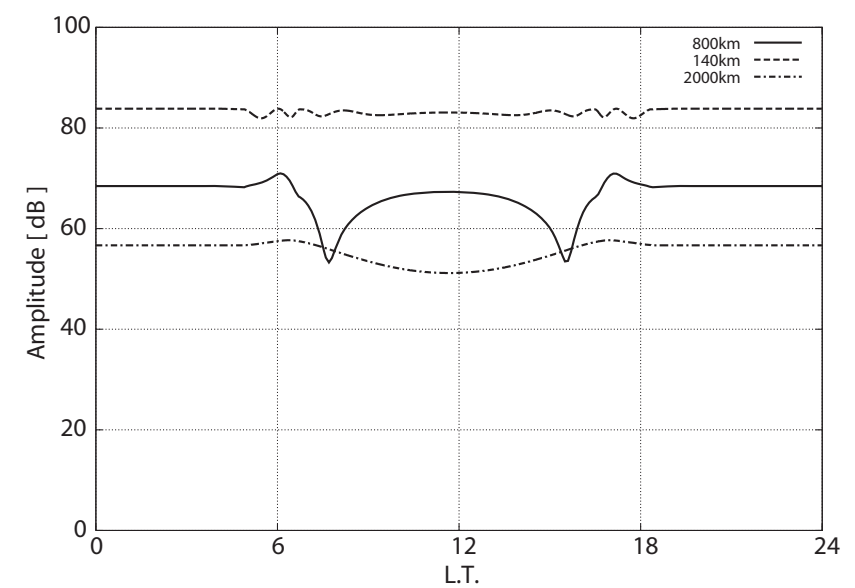

Fig. 6. Diurnal variation of the resultant electric field intensity at $\mathrm{f}=40 \mathrm{kHz}$ (JJY case) with changing the propagation distance $(800$, 140 and $2000 \mathrm{~km})$.

at each station, simultaneously several VLF/LF transmitters; (i) NWC (Australia, $\mathrm{f}=19.8 \mathrm{kHz}$ ), (ii) NPM (Hawaii, $21.4 \mathrm{kHz}$ ), (iii) NLK (USA, $24.8 \mathrm{kHz}$ ), (iv) JJI (Kyushi, Ebino, $\mathrm{f}=22.2 \mathrm{kHz}$ ) and (v) Japanese standard wave transmission, JJY ( $\mathrm{f}=40 \mathrm{kHz}$ ) (in Fukushima prefecture). This VLF/LF network is established for the study of seismoionospheric perturbations (Hayakawa et al., 2004). In the following we pay our main attention to the JJY transmitter, because this transmitter is located approximately in the middle of Japan and the distance from this transmitter to each observing station is at close distances.

Figure 3a illustrates the distance dependence of the electric field intensity in a test direction from JJY (geographic cooridinates: $37^{\circ} 22^{\prime} \mathrm{N}, 140^{\circ} 51^{\prime} \mathrm{E}$ ) to Kochi station (the azimuthal direction from JJY to Kochi station is $242^{\circ}$ from the
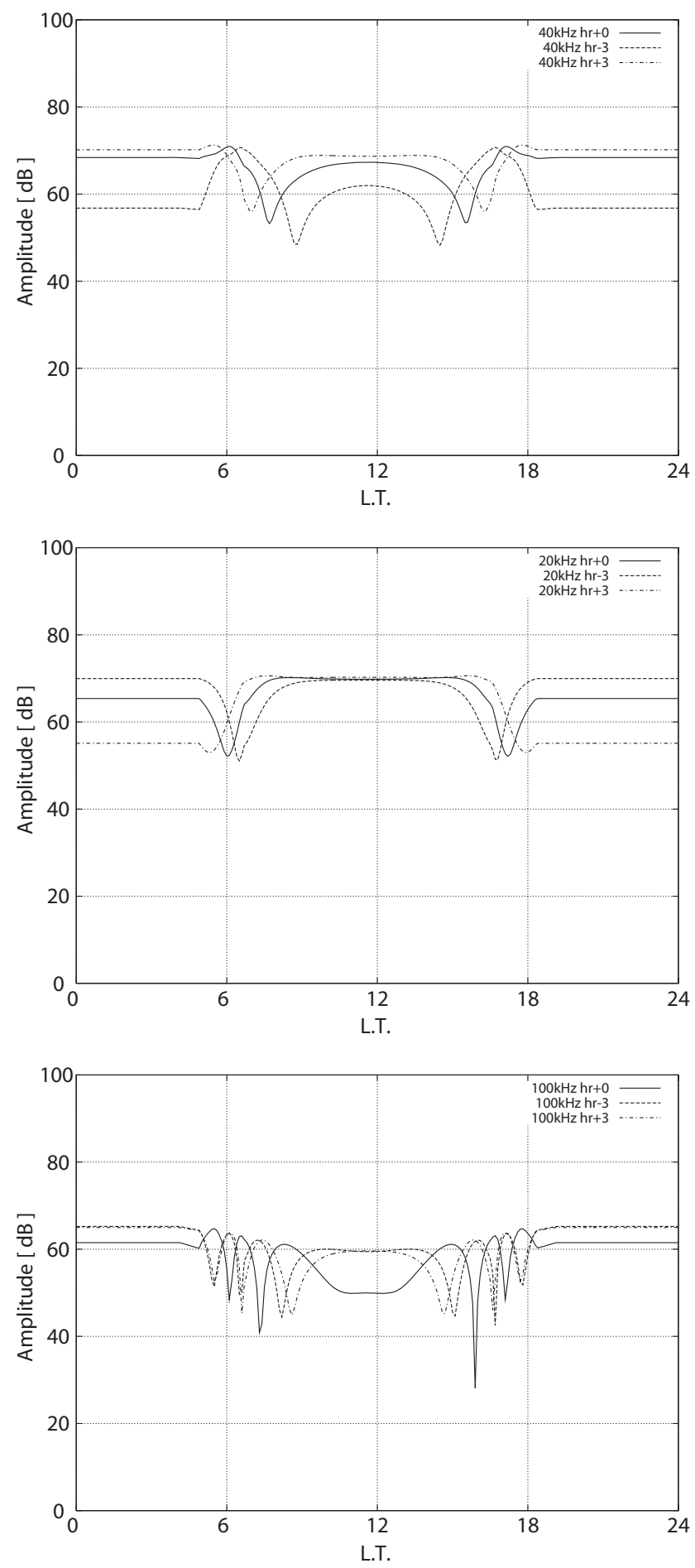

Fig. 7. Effects of shift in ionospheric reflection height on the diurnal amplitude variation. (a) $40 \mathrm{kHz}$, (b) $20 \mathrm{kHz}$ and (c) $100 \mathrm{kHz}$.

North). The Earth's magnetic field effect is also included in this computation. When we specify the location (latitude and longitude) of the mid-point between the transmitter and receiver (in the case of 1-hop ray), relevant month 
and local time, solar activity etc., we can determine the ionospheric profile. So that we are ready to compute the matrix ionospheric reflection coefficients for our relevant frequency and incident direction (incident and azimuthal angles) at the relevant mid-point of reflection, with taking into accout the Earth's magnetic field effect (Budden, 1961). The coefficient of reflection $\mathrm{R}_{i}$ in Eqs. (1), (3) and (4) is approximated by that of ${ }_{\|} R_{\|}$(TM incident, TM reflected) in the matrix ionospheric reflection coefficients. The $\mathrm{R}_{g}$ is computed by the Fresnel coefficient. The frequency of JJY is $40 \mathrm{kHz}$, and its radiation power is $50 \mathrm{~kW}$. The ionospheric height $\mathrm{h}$ is a function of solar zenith angle (Belrose, 1968) and it is about $75 \mathrm{~km}$ at day and $95 \mathrm{~km}$ at night. The coefficients $\mathrm{R}_{i}, \mathrm{~F}_{i}$, and $\mathrm{D}_{g}$ are parameters depending on frequency and distance. For example, $\mathrm{F}_{i} \cong 1.13, \mathrm{~F}_{t} \cong 0.9, \mathrm{~F}_{r} \cong 0.9$ are used in the computations. Figures $3 \mathrm{a}-\mathrm{c}$ are the figures of electric field intensity as a function of distance up to $\mathrm{d}=2000 \mathrm{~km}$. The full line refers to 1-hop wave, the dotted lines, the results for up to 2 hops (i.e. 1-hop+2-hop), and the dot-full lines, those up to 3hop wave. The ground wave is expressed by the broken line in these figures. Figure $3 \mathrm{a}$ refers to the JJY case $(40 \mathrm{kHz})$, while Figs. $3 \mathrm{~b}$ and $3 \mathrm{c}$ correspond to the lower transmission frequencies of $20 \mathrm{kHz}$ and $100 \mathrm{kHz}$, but at the same place of transmission. It is found from a comparison of these figures that the effect of higher-hop sky waves increases with the increase in propagation distance. This is easily understood.

\section{Generation mechanism of terminator times in subionospheric VLF/LF diurnal variation}

We here consider a short-distance propagation condition, in which the transmitter is JJY station (frequency $=40 \mathrm{kHz}$ ) and the receiver is located at Kochi (geographic coordinates; $33.55^{\circ} \mathrm{N}, 133.53^{\circ} \mathrm{E}$ ). So the propagation distance is $\mathrm{d}=786 \mathrm{~km}$. With taking into account the results in the previous section, we have taken into consideration up to 2-hop waves. Figure $4 \mathrm{a}$ is the computed diurnal variation of the electric field intensity at the receiver. The broken line refers to the ground wave, the dot-broken line, to the sky waves, and the full line is the resultant wave. We notice clearly the presence of so-called terminator times with amplitude minima. We will show how these terminator times are formed. As is shown in Fig. 4a, the amplitudes of the ground and sky waves do not exhibit any significant diurnal changes. While, Fig. $4 \mathrm{~b}$ illustrates the corresponding diurnal variations of reflection height and phase delay of only 1-hop sky wave with respect to the ground wave ( $\phi_{1}$ in Fig. 2) (middle panel) (because the 2-hop wave is much weaker than the 1-hop wave, so that it is not considered here). It is found that when the phase delay $\phi_{1}$ is $180^{\circ}$, we have terminator times in the diurnal variation. So that, we can conclude that the terminator time is formed as an interference between the ground wave and sky wave.

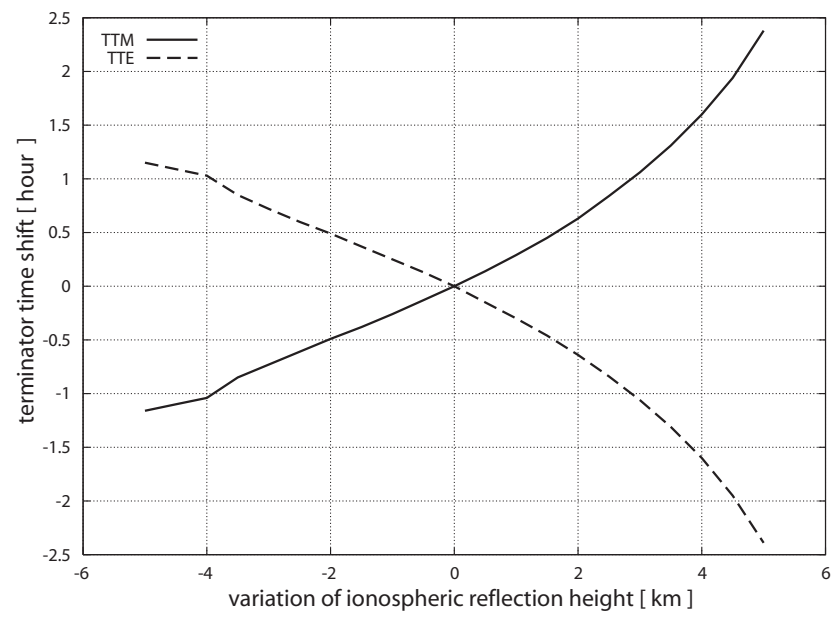

Fig. 8. The relationship between the terminator time shift and the change of ionospheric height. - means lowering, while + indicates upward shift of the lower ionosphere. Both terminator time evening (TTE) and terminator time morning (TTM).

For the fixed propagation distance from the JJY transmitter to Kochi $(\mathrm{d}=786 \mathrm{~km})$, we have changed the frequency and Fig. 5 is the result on the terminator times. At lower frequencies of 20 and $40 \mathrm{kHz}$, we have one minimum around sunrise and sunset, while we have two terminator times around sunrise and sunset for $100 \mathrm{kHz}$, simply due to a smaller wavelength of $100 \mathrm{kHz}$.

While, in Fig. 6 we have fixed the frequency of $40 \mathrm{kHz}$ (JJY case) and for a fixed azimuth from the JJY to Kochi, but we have changed propagation distance (from $800 \mathrm{~km}$ to two values smaller and greater than $800 \mathrm{~km}$ i.e., $140 \mathrm{~km}$ and $2000 \mathrm{~km}$ ). Figure 6 indicates that the terminator time appears very clearly for shorter distances of $800 \mathrm{~km}$. When the distance becomes smaller $(140 \mathrm{~km})$, we can espect some terminator time effect even though the ground wave is much more dominant than the sky wave. But, no terminator time appears for $2000 \mathrm{~km}$.

\section{Application of the terminator time to seismogenic study}

As is already shown by Hayakawa et al. (1996) and Molchanov et al. (1998), the significant shift in terminator time for the Kobe earthquake over a short distance of $\sim 1000 \mathrm{~km}$ was interpreted in terms of a few $\mathrm{km}$ depletion of the lower ionosphere before the earthquake by using the fullwave method. We assume the propagation path from JJY to Kochi. The frequency is widely varied from $40 \mathrm{kHz}$ of our main interest to 20 and $100 \mathrm{kHz}$. The lower ionosphere is shifted in height by $-3 \mathrm{~km},-2 \mathrm{~km},-1 \mathrm{~km}$ (these three, downward shifting), $+1 \mathrm{~km},+2 \mathrm{~km}$ and $+3 \mathrm{~km}$ (upward shifting). Figure 7 illustrates the effect of shift in ionospheric reflection height on the diurnal variation. Figure 7 a refers to 
$40 \mathrm{kHz}$ (JJY case), in which the full line refers to the case of no perturbation and the broken line refers to the lowering the ionospheric and the dot-broken line corresponds to the upward shift of the ionosphere. When the ionosphere is lowered, the morning terminator time shifts to early hours and the evening terminator time to later evening. That is, we can say that the daytime felt by the waves becomes longer. This is in excellent agreement with former experimental results (Hayakawa et al., 1996; Maekawa et al., 2006). The similar tendency can be confirmed for other frequencies in Figs. 7b and $7 \mathrm{c}$.

Finally, we show the quantitative estimation on the relationship between the shift in terminator time (terminator time morning TTM and terminator time evening TTE) and the change in ionospheric height. The transmitter and receiver are fixed to JJY $(\mathrm{f}=40 \mathrm{kHz})$ and Kochi, and Fig. 8 illustrates the corresponding results. When the ionosphere is lowered as in the case of earthquakes (Hayakawa et al., 1996), TTE shifts to later hours and TTM shifts to earlier hours. When $\Delta \mathrm{h}=-2 \mathrm{~km}$, the shifts in both TTE and TTM are found to be of the order of $0.5 \mathrm{~h}(30 \mathrm{~min})$, which are usually observed in the experiments. This figure will enable us to infer the information on the perturbation of lower ionosphere before the earthquake. Hence, short (but not too short) distances (from a few hundred to $2000 \mathrm{~km}$ ) offer a higher sensitivity for detecting the ionospheric modifications including those originating from seismic activity.

\section{Conclusions}

The generation mechanism of terminator times in subionospheric VLF/LF propagation has been studied for relatively short distances (less than $2000 \mathrm{~km}$ ), and it is found that the terminator time is formed as an interference between the ground wave and sky wave. Finally, the relationship between the terminator time shift and change in the ionospheric height, has been investigated, which suggests its possible use in inferring the lowering of the ionosphere before the earthquakes.

Acknowledgements. The authors would like to thank NiCT for its support ( $\mathrm{R}$ and $\mathrm{D}$ promotion scheme funding international joint research).

Edited by: M. Contadakis

Reviewed by: P. F. Biagi and A. Nickolaenko

\section{References}

Belrose, J. S.: Low and very low frequency radio wave propagation, in Radio Wave Propagation, AGARD Lecture Ser., 29, 97115, Advisory Group for Aerospace Research and Development, NATO, France, 1968.

Biagi, P. F., Castella, L., Maggipinto, T., Ermini, A., Perna, G., and Capozzi, V.: Electric field strength analysis of 216 and $270 \mathrm{kHz}$ broadcast signals recorded during 9 years, Radio Sci., 41, RS4013, doi:10.1029/2005RS003296, 2006.

Budden, K. G.: The Wave-Guide Mode Theory of Wave Propagation, Logos Press, London, 325 pp., 1961.

Hayakawa, M. and Molchanov, O. A.: Seismo Electromagnetics: Lithosphere - Atmosphere - Ionosphere Coupling, TERRAPUB, Tokyo, 477 pp., 2002.

Hayakawa, M. and Fujinawa, Y.: Electromagnetic Phenomena Related to Earthquake Prediction, Terra Sci. Pub. Co., Tokyo, 667 pp., 1994.

Hayakawa, M., Molchanov, O. A., Ondoh, T., and Kawai, E.: The precursory signature effect of the Kobe earthquake on VLF subionospheric signals, J. Comm. Res. Lab., Tokyo, 43, 169$180,1996$.

Hayakawa, M.: Atmospheric and Ionospheric Electromagnetic Phenomena Associated with Earthquakes, Terra Sci. Pub. Co., Tokyo, 996 pp., 1999.

Hayakawa, M. and Molchanov, O. A.: NASDA/UEC team, Summary report of NASDA's earthquake remote sensing frontier project, Phys. Chem. Earth, 29, 617-625, 2004.

International Telecommunication Union, Recommendation ITU-R, Ground wave propagation curves for frequencies between $10 \mathrm{kHz}$ and 3 DMHz,Geneva, Switzerland, P. Series, p. 368-7,1992.

International Telecommunication Union, Recommendation ITU$\mathrm{R}$, Prediction of field strength at frequencies below $150 \mathrm{kHz}$, Geneva, Switzerland, P. Series, p. 684-3, 2002.

Maekawa, S., Horie, T., Yamauchi, T., Sawaya, T., Ishikawa, M., Hayakawa, M., and Sasaki, H.: A statistical study on the effect of earthquakes on the ionosphere, based on the subionospheric LF propagation data in Japan, Ann. Geophys., 24, 2219-2225, 2006, http://www.ann-geophys.net/24/2219/2006/.

Molchanov, O. A. and Hayakawa, M.: Subionospheric VLF signal perturbations possibly related to earthquakes, J. Geophys. Res., 103, 17 489-17 504, 1998.

Molchanov, O. A., Hayakawa, M., Ondoh, T., and Kawai, E.: Precursory effects in the subionospheric VLF signals for the Kobe earthquake, Phys. Earth Planet. In., 105, 239-248, 1998.

Rozhnoi, A., Solovieva, M. S., Molchanov, O. A., and Hayakawa, M.: Middle latitude LF $(40 \mathrm{kHz})$ phase variations associated with earthquakes for quiet and disturbed geomagnetic conditions, Phys. Chem. Earth, 29, 589-598, 2004.

Shvets, A. V., Hayakawa, M., Molchanov, O. A., and Ando, Y.: A study of ionospheric response to regional seismic activity by VLF radio sounding, Phys. Chem. Earth, 29, 627-637, 2004.

Wakai, N., Kurihara, N., Otsuka, A., and Imamura, K.: Prediction method of LF/MF field strangths, Inst. Electr. Information Comm. Engrs. Japan, Technical Report, AP-2003-271, 35-40, 2004. 